\title{
Global Regulator MorA affects Virulence-associated Protease Secretion in Pseudomonas aeruginosa PAO1 Supporting Information
}

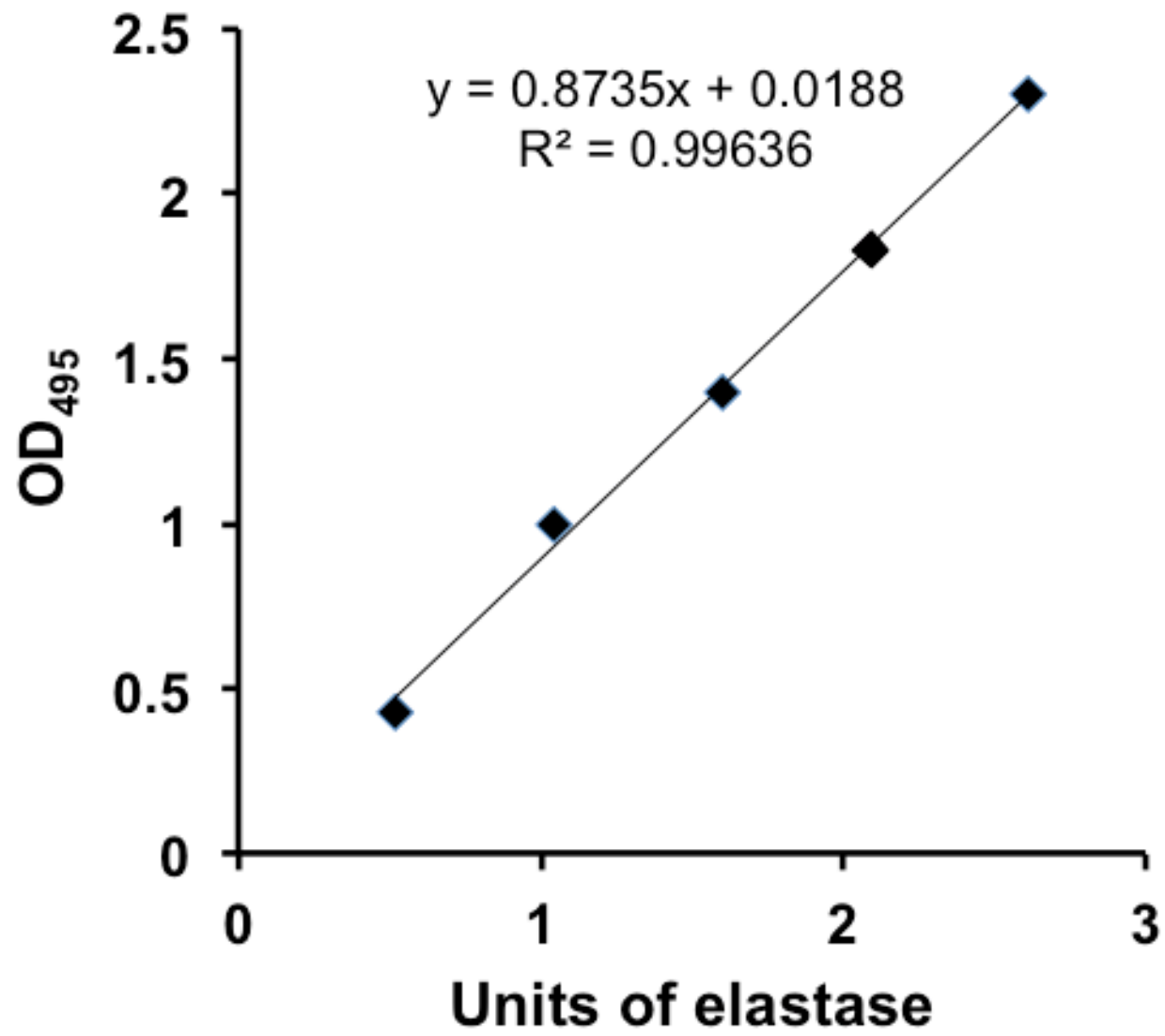

S1 Figure. Standard curve for elastase activity. Activity measured with increasing units of Pseudomonas aeruginosa elastase on elastin congo-red (Sigma). Reaction buffer without elastase was used as blank. At end of $6 \mathrm{hr}$ incubation, the absorbance of the supernatant containing cleaved congo-red was measured at $495 \mathrm{~nm}$. 